\title{
Flexible fiberoptic bronchoscopy
}

\author{
Ramalinga P. Reddy and Pierre A. Vauthy \\ Toledo Hospital Pediatric Pulmonary Centre and the Department of Pediatrics, \\ The Medical College of Ohio at Toledo, U.S.A.
}

\begin{abstract}
Although the availability of flexible fiberoptic bronchoscopy (FFB) has been a major advance in adult pulmonary medicine, the role of FFB in pediatric patients is not well defined. In this article, we try to review our 10-year experience in over 4,000 cases where FFB has been utilised. We feel that this procedure is safe, cost effective and is tolerated much better than the laryngoscopy or open tube bronchoscopy. It is rapid in diagnosing upper airway obstruction and can be done in an upright position.
\end{abstract}

\section{Key words : Flexible fiberoptic bronchoscopy; pulmonary interstitial emphysema}

Open tube bronchoscopy was first introduced to American medicine in the nineteenth century. Routine bronchoscopy was first used in the Mayo Clinic by Henry Plummer as early as 1904 . In 1969 Shigeto Akeda of Japan brought to the Mayo Clinic the first bronchofiberscope-made of fiberoptic materials incorporated into a flexible bronchoscope. The introduction of the fiberoptic bronchoscope has revolutionised the diagnostic capability in adult chest medicine. It is generally agreed that this procedure is safer, more comfortable and in many cases, more informative than rigid bronchoscopy. Thus, the indications for flexible bronchoscopy in adult patients are considerably liberalised. However, its use had been limited until recently to adults because of lack of smaller flexible instruments. With the introduction of

Reprint requests: Dr. R.P. Reddy, Toledo Hospital Pediatric Pulmonary Centre and the Department of Pediatrics, The Medical College of Ohio, Toledo, Ohio 43606, U.S.A. such instruments by the Olympus Company, the smaller pediatric airways are no longer inaccessible.

\section{Material and Methods}

Instruments. Three pediatric prototypes of flexible bronchoscopes have been used. All of them have been developed by the Olympus Company (Olympus Corporation of America, Lake Success, New York): The BF 3 C3 was an earlier model and had only limited use. The external diameter of this instrument is 3.4 $\mathrm{mm}$ and it has a flexible tip. In 1978 the standard model BF 3 C4 was made available. This scope has an external diameter of $3.5 \mathrm{~mm}$ and has a suction channel of $1.2 \mathrm{~mm}$ which can be utilised either to suction secretions or to administer oxygen. This scope is $77 \mathrm{~cm}$ long and the distal $2 \cdot 5 \mathrm{~cm}$ may be flexed from approximately +160 degrees to -60 degrees.

The newer prototype has a $2.7 \mathrm{~mm}$ external diameter. It has a flexible tip but no suction channel. On occasion the 
Olympus BF2, that has an outer diameter of $5.3 \mathrm{~mm}$ has been used in children over five years of age. This instrument has a suction channel of $2 \mathrm{~mm}$.

Patients. Over the last 10 years, from 1975 to 1985 , approximately 4,000 procedures have been performed by one of the authors. About $80 \%$ of the patients are under two years of age. The bronchoscope has been used even in premature infants weighing only $600 \mathrm{gm}$. In general, patients range from premature infants to adolescents.

Setting. All procedures were performed either in an intensive care setting or in a treatment room with the availability of supplemental oxygen, emergency medications, suction and resuscitation equipment. There were always one or two physicians monitoring the patient. The patients had a cardio respiratory monitor as well as a transcutaneous monitor when necessary. The patients, either in the pediatric intensive care unit or in the neonatal intensive care unit, did not have to be moved to a different setting for the procedure.

Only three patients had been in an operating room. After the removal of a foreign body from the patient's airway with a rigid scope, the flexible scope was used to detect the fragments in distal airways that are not easily accessible by the rigid scope.

Anesthesia. Patients fasted for four to six hours before the procedure. Infants do not routinely receive premedication. Older children received intramuscular injection of "lytic cocktail" (Demerol, Phenergan and Thorazine) half an hour prior to the procedure. Infants and child- ren under five years of age are usually restrained by a papoose board and are studied with the head elevated at 40-45 degrees. Older children rarely need restraints. All patients with acute airway obștruction are studied in the upright position. Two per cent xylocaine jelly is inserted into the nasal alae which provides both lubrication and topical anesthesia. Topical cetacaine spray is administered to the oropharynx and larynx if bronchoscopy is contemplated. Over the vocal cords, $1 \%$ xylocaine liquid is instilled through the suction channel of the bronchoscope.

Procedure or technique. Transnasal examination is preferred. This is performed by inserting the bronchoscope into either nares and passing it along the floor of the nose. Once the nasopharynx is reached, flexion of the tip and further insertion allowed visualisation of the base of the tongue, vallecula, epiglottis and larynx. Laryngeal structures and function are then observed from above. In order to adequately evaluate the dynamics of the upper airway, it is often necessary to observe the laryngeal structures and airway in prone as well as in supine position. Sometimes sedation with Demerol is given to evaluate structures in a fully relaxed state. When examination of tracheobronchial tree is contemplated, the vocal cords are sprayed with $1 \%$ xylocaine solution and the bronchoscope is advanced through the vocal cords while at the same time the tip of the scope is gently straightened. Sometimes it is necessary to instil xylocaine solution into the tracheobronchial tree in older patients for adequately study.

All the procedures done in the treatment room are videotaped by the Circon 
Color video system. This allows for immediate playback, documentation and teaching purposes. The procedures done either in an intensive care setting or in the emergency department are watched simultaneously by another examiner or observer with the aid of the teaching attachment (Olympus LS-2). In addition, the structures and their abnormalities have often been photographed with an adaptable camera-Olympus SC 16. This helps not only in validating our observations but also aids teaching.

Transoral examination is performed in infants with abnormalities of nasopharynx such as nasal polyps, severe choanal stenosis or choanal atresia by passing the bronchoscope over the surface the tongue. When once the posterior pharynx is reached, flexion of the tip of the bronchoscope enables us to view the epiglottis and larynx. Sometimes, in both transoral and transnasal approach, the epiglottis may fall back posteriorly in a fully relaxed state and make it difficult to evaluate the vocal cords and the subglottic space. By turning the patient to the side or by making the patient sit upright visualisation of laryngeal structures is made easier.

Often the bronchoscope has been passed through the endotracheal tube in patients who have been intubated. In these patients, the oxygen has been increased to $100 \%$ prior to and during the procedure. With an adaptor, the bronchoscope is then advanced through the endotracheal tube while the patient is still attached to the ventilator. This helps us evaluate the abnormalities of the lower airway.

On occasion, the bronchoscope has also been passed either through a tracheostomy tube or tracheostomy opening to study the lower airway structures and to suction selective airways. One can also evaluate the subglottic space and vocal cords from below during this procedure.

In open tube or rigid bronchoscopy the patient can breathe through the bronchoscope. However, in flexible bronchoscopy the patient has to breathe around the bronchoscope. The trachea of a full term newborn infant is approximately $5 \mathrm{~mm}$ in diameter. With $3.5 \mathrm{~mm}$ bronchoscope in the trachea it would be difficult for the infant to breathe around it. We limit the examination of lower airways to 30 or 45 seconds. Often, after the bronchoscope passes through the glottis, the examination of all segments and lobes can be accomplished in 30 seconds. When there are large quantities of mucous secretions in the airway, we have used the method of flushing oxygen through the suction channel to enable us to evaluate the airway. We can also ventilate the patient this way and we have documented this by using transcutaneous oxygen and carbon dioxide monitors. Adequate gas exchange can take place for several minutes. However, it is necessary in all cases to monitor patients closely and to complete evaluation as rapidly as possible to avoid hypoxia and hypercarbia.

Indications (Table 1). In general, indications for FFB in infants and children are the same as those for rigid bronchoscopy. Since the FFB can be used in an outpatient setting and as this procedure has been shown to be safe and rapid, the indications for flexible fiberoptic bronchoscopy have been liberalised. Chevalier Jackson, one of the pioneers in the field of bronchoscopy, once said, "In case of doubt as to whether bronchoscopy should be done or not, bronchoscopy should always be done." 
Table I. Indications for airway evaluation

Stridor
Wheezing
Persistant atelectasis
Tracheostomy evaluation
Evaluation of subglottic space in patients with
prolonged intubation
Airway compression
Nonspecific X-ray findings
Suspected foreign body aspiration
Cough
Hemoptysis
Hoarseness

\section{Discussion}

In our own center the FFB is utilised even for routine intubations. Nasotracheal intubation can easily be accomplished with the aid of flexible scope. The FFB is first inserted through the nares with the endotracheal tube around it. When once the scope is passed into the trachea, the endotracheal tube is advanced over the scope and then the scope is withdrawn thus intubating the patient nasotracheally. We feel that this method of intubation is safe, far better tolerated, less traumatic and rapid. One can also see the position of the distal end of the endotracheal tube. Although radiologic confirmation is necessary for documentation and evaluation of lung, the examination can be delayed if necessary. Intubation with a scope is often necessary in patients who are suspected to have cervical trauma. With different scopes, endotracheal tubes from size 3 onwards are used. Also, in children with severe acute upper airway obstruction, the scope has been utilised to establish an airway. Over the last 10 years 40 patients with acute epiglottitis underwent intubation wirh the scope without anesthesia. They have tolerated the procedure well and no mortality or morbidity was recorded.

In patients with membranous tracheitis, in addition to establishing the airway, the flexible scope has been very useful to dislodge thick, tenacious, membranous secretions from the trachea and relieve acute airway obstruction.

The FFB has been particularly useful in selective intubation of one of the main stem bronchi. In many cases of pulmonary interstitial emphysema with unilateral pseudocyst formation and herniation of the lung with mediastinal shift, it is often necessary to selectively intubate the contralateral main stem bronchus. The flexible instrument is just suited for that. The flexible instrument has also been used in an older child for selective intubation of left main stem bronchus. In an illustrative case history, NA, a four-yearold child underwent right pneumonectomy for pulmonary sequestration involving the right lung. The diagnosis was significantly delayed and by the time of pneumonectomy, the patient had significant pulmonary hypertension and enlarged pulmonary arteries. After right pneumonectomy with mediastinal shift to the right side, the left main stem bronchus was compressed by the left pulmonary artery. The patient required selective intubation of the left main stem bronchus with the flexible scope for a prolonged period of time. The patient's hospital course was complicated by the development of polyps in the left main stem bronchus distal to the endotracheal tube, most likely caused by the chronic irritation of suction catheters. The endotracheal tube needed to be advanced on several occasions which was again accomplished with the FFB. Ultimately after reinforcing the left main stem bronchus with a cartilaginous 
graft, the patient was successfully decannulated and has been doing well. For this procedure, the patient required cardiopulmonary bypass.

The flexible scope is invaluable in evaluating laryngeal function. With the transnasal approach, one can study the function of laryngeal structures without distorting the normal anatomy. Laryngel function can be studied for a long time in various positions and also in an awake state as well as in a fully relaxed state. Impingement upon the epiglottis by enlarged and posteriorly extending tonsils can be evaluated by the scope. In some cases, stridor could be from posterior extension of the base of the tongue or from collapsing lateral pharyngeal walls. This can be well-evaluated with the transnasal approach with the head and neck in natural neutral position.

The bronchoscope is used to evaluate anterior compression of the trachea as is seen in anomalous innominate artery compression. The conventional barium swallow will not usually show extrinsic anterior tracheal compression. However, with the flexible scope it is easier to see the oblique or oval appearance of trachea at the site of compression.

In the evaluation of tracheoesophageal fistula, especially in a newborn, the flexible scope is utilised. The surgeon can simultaneously evaluate the airway and confirm the findings of TE fistula with the aid of the teaching head. One can avoid a barium swallow and possible risk of aspiration of barium into the tracheobronchial tree. In the evaluation of H-type TE fistula, we have used the "methylene blue" study. In this procedure, with the tip of the bronchoscope in the trachea below the level of glottis, a nasogastric tube is inserted into the esophagus and a small amount of dilute methylene blue dye is injected into the esophagus. One can see the dye entering the trachea through the small H-type TE fistula. This was successful in two cases when three times contrast studies with barium and lower viscosity gastrograffin failed to show the fistula.

Thus, the practical uses of the bronchoscope are many. It is often used in selective bronchography, recurrent atelectasis, not only to suction secretions but to insufflate the lung as well.

Complications. The frequency of complications has been very low. These could be divided into major and minor. In five patients, transient epistaxis followed the procedure. None required any treatment. One patient experienced transient laryngospasm following the application of cetacaine spray to the larynx. This resolved spontaneously and did not require intubation. Transient bradycardia was seen in several infants and premature infants. Two patients required atropine. Major complications included six instances of pneumothorax. These occurred in infants of less than three months of age with significant lung disease. Bronchoscopy was done because of persistent atelectasis. Penumothorax followed insufflation of the lung. Two of these infants required chest tubes.

Methemoglobinemia was seen in one three-year-old child after the application of cetacaine spray.

Advantages of $F F B$ over rigid scope. FFB, as opposed to open tube bronchoscopy, can be done in an outpatient setting. It is cost-effective and the risk of general anesthesia is avoided. This is well exemplified in a child with cough after choking while eating food. When an 
aspiration of foreign body is suspected with normal X-rays, flexible bronchoscopy is performed as an outpatient. If no foreign body is found, the patient is sent home. If there is a foreign body, the patient is admitted to the hospital for removal of FB under anesthesia with a rigid scope. Subglottic swelling is rarely seen with FFB, unlike rigid bronchoscopy. FFB can be done with a patient in an upright position. This is again advantageous in a patient suspected to have acute upper airway obstruction from acute epiglottitis. This can be done in an ICU setting as well as in the emergency department. The function of laryngeal structures can be evaluated in greater detail without distorting the anatomy. It can be utilised even in smaller, premature infants and airways distal to main stem bronchii can also be evaluated. It has been used to intubate patients in diffiult cases.

Disadvantages. For practical purposes, the flexible bronchoscope is solid and the patient has to breathe around it while he can breathe through the rigid scope. A child with a $5 \mathrm{~mm}$ trachea has a difficult time ventilating with a $3.5 \mathrm{~mm}$ scope in place. It is also difficult to adequately evaluate the subglottic space with FFB unless the observer is extremely experienced. FFB is difficult to perform in an uncooperative and combative child. Also, the FFB cannot be routinely used in the removal of foreign bodies from the airways. An unskilled examiner may even push the foreign bodies distally with FFB.

The quality of the optical image obtained through the flexible scope is inferior to the one obtained through rigid scope equipped with a glass rod telescope. Thus still photography with a rigid scope is superior.
In this short article, we tried to review our experience with flexible bronchoscope in pediatrics. We find the technique to be safe, rapid, cost-effective and many times more informative. The smaller pediatric airways are more accessible with the flexible scope. The experience of other endoscopists many be different. In this review we are only presenting our personal point of view based on our experience.

\section{References}

1. Jackson C. Peroral endoscopy and laryngeal surgery. The Laryngoscope Co., 1915, St. Louis, Mo. 201

2. Fan LL, Flynn JW. Laryngoscopy in neonates and infants : experience with flexible fiberoptic bronchoscopy. Laryngoscope 1981; $91: 451-456$

3. Fitzpatrick SB, Marsh B, Stokes KD, et al. Indications for flexible fiberoptic bronchoscopy in pediatric patients. Am $\mathbf{J}$ Dis Child 1983; $137: 595-597$

4. Holinger LD. Etiology of stridor in the neonate, infant and child. Ann Otol 1980; 89 : $397-400$

5. Nussbaum E. Flexible fiberoptic bronchoscopy and laryngoscope in infants and children. Laryngoscopy $1983 ; 93: 1073-1075$

6. Rucker RW, Silva WJ, Worcester CC. Fiberoptic bronchoscopy nasotracheal intubation in children. Chest $1979 ; 76: 56-58$

7. Silberman HD. The use of the flexible fiberoptic nasopharyngoscope in the pediatric upper airway. Otolaryngol Clin North Am 1978; $11: 365-370$

8. Vauthy PA, Reddy R. Acute upper airway obstruction in infants and children, evaluation by the fiberoptic bronchoscope. Ann. Otol. Rhinol. Laryngology $1980 ; 89: 417-418$

9. Vigneswaran R, Whitfield JM. The use of a new ultrathin fiberoptic bronchoscope to determine endotracheal tube position in the sick newborn infant. Chest 1981; 80 : 174177

10. Wood RE, Fink RJ. Applications of flexible fiberoptic bronchoscopies in infants and children. Chest $1978 ; 73: 737-740$ 
11. Wood RE, Sherman JM. Pediatric flexible bronchoscopy. Ann. Otol. Rhinol. Laryngol $1980 ; 89: 414-416$

12. Wood RE. Spelunking in the pediatric airways : Explorations with the flexible fiberoptic bronchoscope. Pediatr Clin North Am 1984; 31 : 785-799

13. Wood RE. The diagnostic effectiveness of the flexible bronchoscope in children. Ped Pulmonology $1985 ; 1: 4-7$

14. Prickett C, LeGrand P. Complications of flexi- ble fiberoptic bronchoscopy in a community hospital. Alabama Med 1984; 53 : 25-27

15. Sheldon RL. Flexible fiberoptic bronchoscopy. Primary Care $1985 ; 12 ; 2$ : 299-315

16. Flower CDR, Shneerson JM. Bronchography via the fiberoptic bronchoscope. Thorax 1984; $39: 260-263$

17. Harada K, Mutsuda T, Saoyama N. Reexpansion of refractory atelectasis using a bronchofiberscope with a balloon cuff. Chest $1984 ; 6: 725-728$ 